\title{
COMPUTER SIMULATION OF ROUNDABOUTS
}

\author{
Tamás Kovács ${ }^{1 *}$ \\ ${ }^{1}$ Department of Information Technology, GAMF Faculty of Engineering and Computer Science, John von \\ Neumann University, Hungary \\ https://doi.org/10.47833/2020.3.CSC.003
}

\begin{abstract}
Keywords:
traffic-simulator

roundabout
\end{abstract}

\section{Article history:}

Received 29 Sept 2020

Revised 18 Oct 2020

Accepted 25 Oct 2020

\begin{abstract}
In the last decades numerous traffic lamp controlled intersections have been changed to roundabouts on Hungarian traffic roads, hoping that this helps diminish the usual traffic jams in these traffic contexts. The most characteristic parameter of a roundabout is the capacity that is the number of vehicles can pass through the intersection in 1 hour. The capacity calculation of the new roundabouts, beyond a formula based calculation, often involves computer simulation as well, so as to get more reliable results. In the present paper we introduce a development of our traffic simulator so that it is able to determine the capacities of a roundabout. The simulation is tested by capacity calculation of a real roundabout and the dependency of the capacity on some key parameters is examined as well.
\end{abstract}

\section{Introduction}

Recently the role of computer simulators in traffic engineering has been growing gradually as more and more reliable and easy-to-use simulators appeared in the software market. These simulators give us cheap and quick answers about new intersections or urban traffic systems prior to expensive experiments and implementations. The roundabouts, being quite complex systems, are especially good objects of simulations. In the case of such intersections usually there is no programmed traffic light control, the capacities (i.e. the highest possible traffic currents for specific directions) are determined by the geometry of the roundabout, and the behavior of the drivers.

There are several mathematical models helping the capacity calculations for roundabouts. One part of these models formulates the highest possible (or average) vehicle speeds that belongs to the path-curves of the intersection. The other part describes the general behavior of the drivers waiting to enter the roundabout. Generally, the relation between the planned path-curves and maximum speed values are formulated by the national traffic planning guides or rules. So these rules can be somewhat different even in the European countries. Some of these rule-collections are published, see for example [1] (USA) or [2] (UK). However, researchers of the field analyzed the proposed speed calculation rules as well.

In 2011 Bassani and Sacchi [3] have developed an empirical model for estimating the roundabout circulating speed as a function of the internal circle diameter, the lane-width of the circular road and the width of the entry lanes. Al-Omari et al. [4] refined further this empirical geometric model with the parameters of the entry angles and the so called drive curve parameter.

Šurdonja et al. [5] recently gave a detailed analysis of the most frequently applied mathematical methods comparing their results to measurements of several benchmark roundabouts. In [6] Akcelik summarized the optimal (negotiating) speed values in table as a function of the diameter of the central circle and the lane-widths and introduced a geometric delay model that considers also the ratio of the stopped and non-stopped vehicles at the approaching roads.

\footnotetext{
* Corresponding author. Tel.: +36 76516412 ; fax: -

E-mail address: kovacs.tamas@gamf.uni-neumann.hu
} 
There are several speed and capacity-calculator software capable to simulate roundabouts. The simulator SIDRA employs deterministic mathematical models, while the systems RODEL and ARCADY use also empirical formulae. The other type is the so-called traffic micro-simulator that models each vehicle based on car-following and driver behavior equations. These equations contains also stochastic elements since the individual driver behaviors are probabilistic in nature. The most known such a system is the VISSIM simulator, which is able to simulate not only roundabouts but a complex traffic contexts with different type of intersections and roads. The VISSIM handles the problem of speed-calculation in a unique way: does not calculates the maximum possible speed value for the curved road-segments, but leaves it to the VISSIM programmer to decide and set the average and maximal speed values for each segments. A detailed overview about these simulators and their capabilities can be read in [7].

Recently the author of the paper participated in developing a traffic micro-simulator that is based on the Intelligent Driver Model [8], and has similar capabilities to those of VISSIM. This simulator was validated by measurements at traffic-lamp controlled intersections [9]. In this paper a development work is introduced that makes it possible to apply this self-developed software for simulating roundabouts accurately. Only this self-developed simulator is used in this work. In the next section the main steps of the applied mathematical modelling are described. In Section 3 the simulation test, which is based on the geometry of a real roundabout, is introduced; and in the final section a brief conclusion of the work is given.

\section{The mathematical model}

In the speed calculation almost all of the mathematical models relies on the dynamic equilibrium equation on a zero elevation road-segment with curve-radius $\mathrm{R}$ :

$$
a_{c p}=\frac{v^{2}}{R}=\mu g
$$

where $a_{c p}$ is the centripetal acceleration, $v$ is the tangential speed, $g=8.81 \mathrm{~m} / \mathrm{s}^{2}$ and $\mu$ is the side friction coefficient. This leads to:

$$
v_{\max }(\text { in } K m / h)=\sqrt{127 R \mu_{\max }}
$$

The maximal values of the side friction are discussed by Akcelik in [6]; for a one-ton motor-car it is approximately 0.21 . Thus, it seems that if the path-segment's inscribed radius is known then the maximal (i.e. 0.85 percentile) and mean speed values are determined. Unfortunately, this is true only for traditional (i.e. not roundabout) intersections, because the central lane-width in a roundabout give possibility to the drivers to use different (optimal) paths on the same road-segment, depending on their turn direction. Similar to VISSIM, our traffic simulator is based on the link-connector model, that is, the road-segments (links) are connected by curved connector paths inside the intersections. Though this model is more detailed than the simple link-node based models, this difference in the optimal paths causes difficulties in simulating a roundabout. Figure 1 shows an illustration of this problem. 


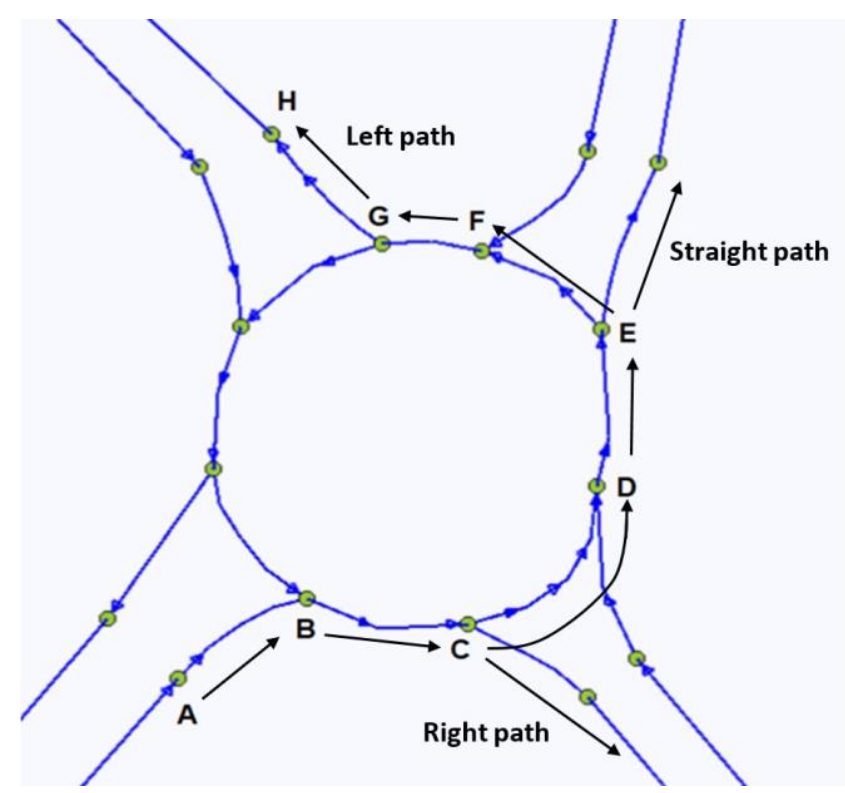

Figure 1. Illustration of a link-connector modelled roundabout and the possible drive-through paths of the vehicles

For example, all vehicles arriving from south-west drives through the connector-endpoints $A$, $\mathrm{B}$ and $\mathrm{C}$, but their path curvature, and hence the inscribed circle radius, are different. Similarly, between points $C, D$ and $E$ the straight-through driving and the left turning vehicles use different trajectories.

In our model the main deficiency is that the lane-width parameter is not considered in determining the maximum speeds. In the previous version of our simulator, the maximum speed value for a connector or road segment was determined by automatically calculating the smallest inscribed circle-radius and applying formula (2). In order to handle roundabouts, the maximum (i.e. 0.85 percentile) speed should be the function of not only the smallest inscribed circle but also the drive-through directions of the vehicles at hand, so, technically, the simple $v_{\max }$ (smallest_radius) function is replaced by the $v_{\max }$ (drive_direction) function. This replacement seems to be a simple task, however, requires a much more complex development work, since the parameters of the roadsegments are static and known before the simulation, while the drive_direction parameter (i.e. right, straight or left) must be determined at the moment of vehicle's arrival to the intersection for each vehicle. Similarly to VISSIM the maximum speed values were calculated a priory by determining the inscribed circle radii of the specific roundabout and using the tabulated speed values published by Akcelik in [6].

Beside path geometry, the other important parameter is the so called critical gap time. If the following time, which is the distance between two consecutive cars on the circular road divided by the velocity, is greater than the critical gap, then a unit car can drive onto the circular road from the approaching road, otherwise not. The value of this parameter is not universal, but can be different for every roundabout. Its value is between 2.0 and 4.0 seconds.

\section{Test of the simulator}

The Figure 2 shows the satellite photo of the roundabout used for the test (Kecskemét Vízmü u.-III.Béla krt.). With the help of the most frequent paths of the vehicles, which can be seen on the photo, the inscribed circles were determined and drawn (by yellow lines on the figure). The error of determining the radii is approximately $\pm 10 \%$, so the approximated error in determining the speedvalues is $\pm 3,3 \%$, since the speed is proportional to the square-root of the radius. The values of $8 \mathrm{~m}$ and $4.5 \mathrm{~m}$ were used for the central lane width and the approaching lane width, respectively. 


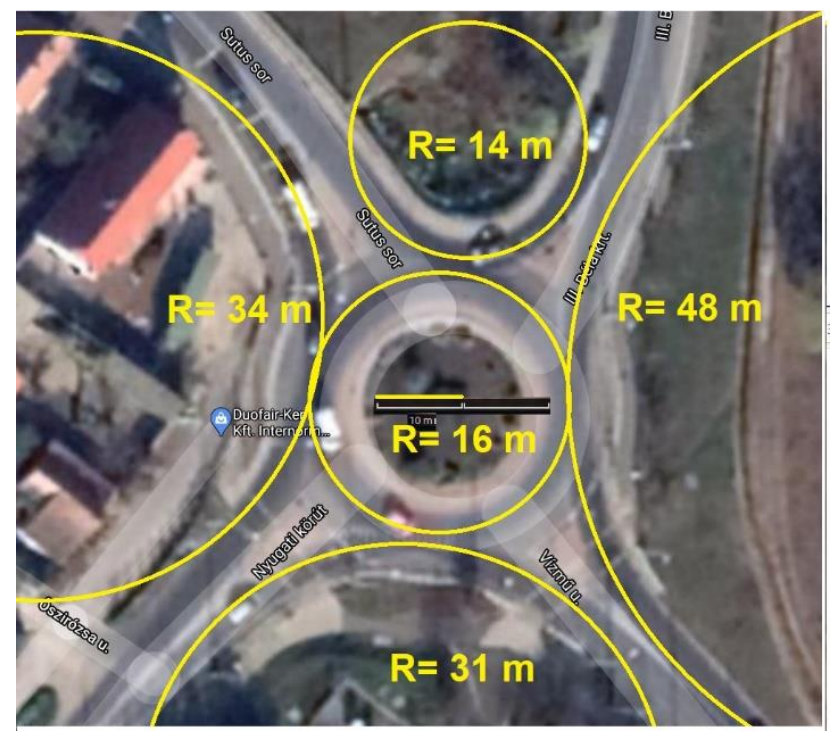

Figure 2. The satellite photograph (by Google) of the roundabout used for the test. The inscribed circles of the most often used paths are drawn by yellow lines.

Based on this geometry the applied average optimal-path speed values are shown in Table 1.

Table 1. The highest safe speed values for the test roundabout

\begin{tabular}{|l|l|}
\hline \multicolumn{1}{|c|}{ Directions } & \multicolumn{1}{c|}{ Highest safe speed } \\
\hline North to West & $28 \mathrm{~km} / \mathrm{h}$ \\
\hline East to North & $18 \mathrm{~km} / \mathrm{h}$ \\
\hline South to East & $34 \mathrm{~km} / \mathrm{h}$ \\
\hline West to South & $27 \mathrm{~km} / \mathrm{h}$ \\
\hline All straight-through & $36 \mathrm{~km} / \mathrm{h}$ \\
\hline All left turning & $21 \mathrm{~km} / \mathrm{h}$ \\
\hline
\end{tabular}

In the test these speed-values and the critical gap values of 2.0; 3,0 and 4.0 secs were used. Only the east-west straight-trough route capacity was examined with traffic volumes in the range of [100 .. 400] vehicles/hour. The only one traffic flow other than that was the south-north (i.e. conflicting) traffic with volume 500 vehicles/hour. The measured quantity was the average travel time through the roundabout on the route at hand as the function of the traffic volume (we call this function as delay curve). The results are shown in Figure 3. The three delay curves drawn by continuous lines correspond to the three critical gap values, and the delay curve drawn by dashed line corresponds to the critical gap value of $3.0 \mathrm{sec}$ but with mean speeds 10 percent higher than the values in Table 1. 


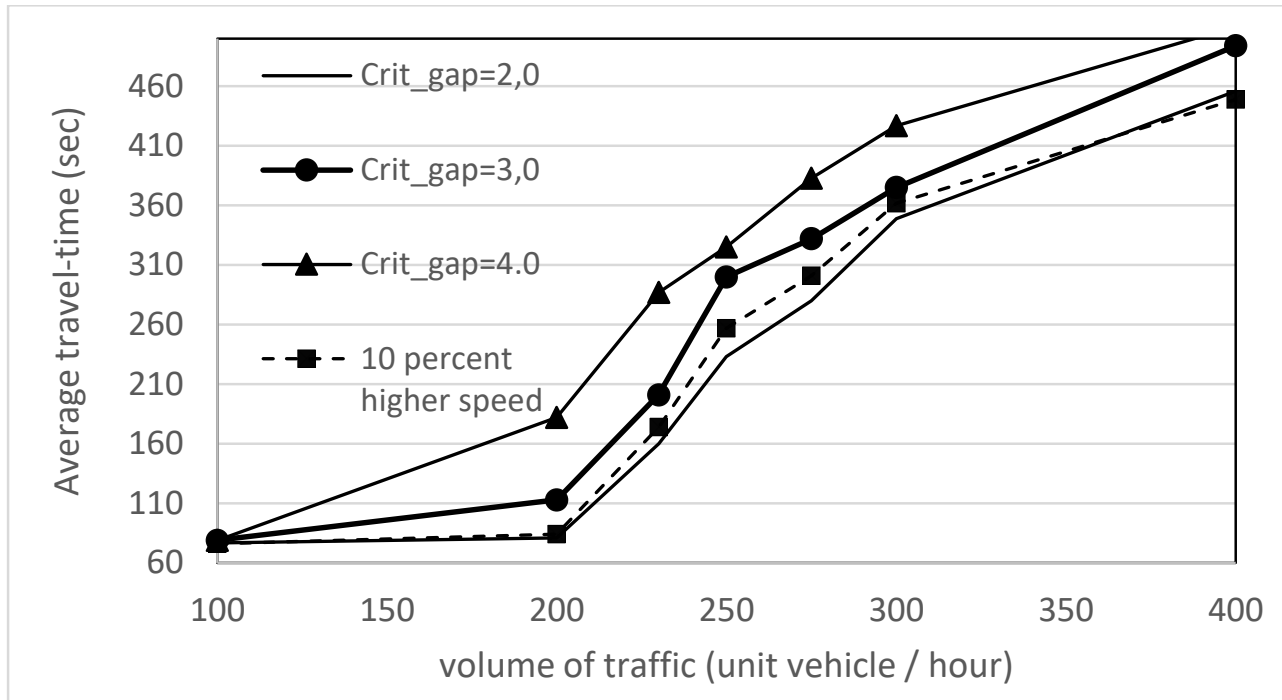

Figure 3. The delay curves of one drive through route of the test roundabout.

The capacity of the intersection in this direction is often considered as the inflexion place of the delay curve, or the half-value place between the lower and the higher plateau. Using the former definition the capacity values in the examined cases are shown in Table 2 . It seems that the capacity value is much more sensitive to the critical gap parameter than to the speed-values, so the $\pm 3,3 \%$ speed determination error can be considered as negligible. On the other hand the critical gap value belongs to this specific roundabout is a much more important parameter.

Table 2. Measured capacity values for the examined cases

\begin{tabular}{|l|l|}
\hline \multicolumn{1}{|c|}{ Case } & Capacity value $($ veh $/ h)$ \\
\hline Critical gap $=2.0 \mathrm{sec}$ & $245 \pm 5$ \\
\hline Critical gap $=3.0 \mathrm{sec}$ & $230 \pm 5$ \\
\hline Critical gap $=4.0 \mathrm{sec}$ & $210 \pm 5$ \\
\hline $\begin{array}{l}\text { Critical gap }=3.0 \mathrm{sec}, \text { and } \\
10 \% \text { higher mean speeds }\end{array}$ & $240 \pm 5$ \\
\hline
\end{tabular}

\section{Conclusion and future work}

Based on the development work and the test measurements we can conclude the followings:

- a link-connector type microscopic traffic simulator can be used for correctly simulating roundabouts by using multiple optimal speed values for the path segments depending on the routes of the vehicles;

- the inscribed circle radii can be determined based on the google satellite photos with an acceptable error;

- the route capacity values are much more sensitive to the value of the critical gap than that of the speed limit.

The test of the development work introduced above can be efficient only if there will be real traffic volume, and so capacity, measurements for comparison. In the following paper such a validating test will be introduced.

\section{Acknowledgment}

This research is supported by EFOP-3.6.1-16-2016-00006 "The development and enhancement of the research potential at John von Neumann University" project. The Project is supported by the Hungarian Government and co-financed by the European Social Fund. 


\section{References}

[1] US Department Of Transportation Contents : Roundabouts-An Informational Guide, https://www.fhwa.dot.gov/publications/research/safety/00067/00067.pdf (Accessed 6 October 2020)

[2] Highways Agency (HA). Geometric Design of Roundabouts. Design Manual of Roads and Bridges. Available at: https://www.standardsforhighways.co.uk/prod/attachments/2b5901c6-3477-4826-b780-cf99003fb5e0 (Accessed 6 October 2020).

[3] Bassani, M. and E. Sacchi. "Experimental Investigation into Speed Performance and Consistency of Urban Roundabouts: an Italian Case Study." Poster Session at the "3rd International Conference on Roundabouts" Transportation Research Board, Carmel, Indiana, USA, May 2011.

[4] Al-Omari, Bashar H., Khalid A. Ghuzlan, and Lina B. Al-Helo. "Modeling through traffic speed at roundabouts along urban and suburban street arterials." Journal of the Transportation Research Forum. Vol. 53. No. 1424-2016117957. 2014., DOI: 10.22004/ag.econ.207432

[5] Šurdonja, Sanja, Vesna Dragčević, and Aleksandra Deluka-Tibljaš. "Analyses of maximum-speed path definition at single-lane roundabouts." Journal of traffic and transportation engineering (English edition) 5.2 (2018): p. 83-95., https://doi.org/10.1016/j.jtte.2017.06.006

[6] Akcelik, R. "Estimating negotiation radius, distance and speed for vehicles using roundabouts. Akcelik \& Associates Pty Ltd." 24th Conference of Australian Institutes of Transport Research. 2004.

[7] Gallelli, Vincenzo, and Rosolino Vaiana. "Roundabout intersections: evaluation of geometric and behavioural features with VISSIM." Proceedings of TRB National Roundabout Conference. Kansas City, Missouri, USA. 2008.

[8] Kesting, Arne, Martin Treiber, and Dirk Helbing. "Enhanced intelligent driver model to access the impact of driving strategies on traffic capacity." Philosophical Transactions of the Royal Society A: Mathematical, Physical and Engineering Sciences 368.1928 (2010): 4585-4605., https://doi.org/10.1098/rsta.2010.0084

[9] Kovács, T., Bolla, K., Alvarez Gil, R., Csizmás, E., Fábián, Cs., Kovács, L., Medgyes, K., Osztényi, J., Végh, A., 2016, Parameters of the intelligent driver model in signalized intersections, Technical Gazette, 23, No. 5, 1469-1474., https://doi.org/10.17559/TV-20140702174255 